\title{
Anne LEGARÉ
}

Professeure associée, Département des sciences politiques, UQÀM

(2012)

\section{“Canada-Québec: un seul État}

\section{et une double diplomatie.”}

Un document produit en version numérique par Jean-Marie Tremblay, bénévole, professeur de sociologie retraité du Cégep de Chicoutimi

Courriel: jean-marie tremblay@uqac.ca

Site web pédagogique : http://www.uqac.ca/jmt-sociologue/

Dans le cadre de: "Les classiques des sciences sociales" Une bibliothèque numérique fondée et dirigée par Jean-Marie Tremblay, professeur de sociologie au Cégep de Chicoutimi Site web: http://classiques.uqac.ca/

Une collection développée en collaboration avec la Bibliothèque Paul-Émile-Boulet de l'Université du Québec à Chicoutimi

Site web: http://bibliotheque.uqac.ca/ 


\section{Politique d'utilisation de la bibliothèque des Classiques}

Toute reproduction et rediffusion de nos fichiers est interdite, même avec la mention de leur provenance, sans l'autorisation formelle, écrite, du fondateur des Classiques des sciences sociales, Jean-Marie Tremblay, sociologue.

Les fichiers des Classiques des sciences sociales ne peuvent sans autorisation formelle:

- être hébergés (en fichier ou page web, en totalité ou en partie) sur un serveur autre que celui des Classiques.

- servir de base de travail à un autre fichier modifié ensuite par tout autre moyen (couleur, police, mise en page, extraits, support, etc...),

Les fichiers (.html, .doc, .pdf, .rtf, .jpg, .gif) disponibles sur le site Les Classiques des sciences sociales sont la propriété des Classiques des sciences sociales, un organisme à but non lucratif composé exclusivement de bénévoles.

Ils sont disponibles pour une utilisation intellectuelle et personnelle et, en aucun cas, commerciale. Toute utilisation à des fins commerciales des fichiers sur ce site est strictement interdite et toute rediffusion est également strictement interdite.

L'accès à notre travail est libre et gratuit à tous les utilisateurs. C'est notre mission.

Jean-Marie Tremblay, sociologue

Fondateur et Président-directeur général, LES CLASSIQUES DES SCIENCES SOCIALES. 
Cette édition électronique a été réalisée par Jean-Marie Tremblay, bénévole, professeur de sociologie au Cégep de Chicoutimi à partir de :

à partir de :

Anne Legaré

“Canada-France : un seul État et une double diplomatie”.

Un chapitre publié dans l’ouvrage sous la direction de Greg Donaghy et Stéphane Roussel, Mission Paris. Les ambassadeurs du Canada en France et le triangle Ottawa-Québec-Paris, chapitre VIII, pp. 163-176. Montréal : Les Éditions Hurtubise, 2012, 216 pp. Collection : Cahiers du Québec, science politique.

[Autorisation formelle accordée par l'auteur le 15 avril 2012 de diffuser ce livre dans Les Classiques des sciences sociales.]

Courriel : legare.anne@uqam.ca

Polices de caractères utilisée : Times New Roman, 12 points.

Édition électronique réalisée avec le traitement de textes Microsoft Word 2008 pour Macintosh.

Mise en page sur papier format : LETTRE US, 8.5'” x 11',

Édition numérique réalisée le 19 avril 2012 à Chicoutimi, Ville de Saguenay, Québec.

\section{Fait avec}

Macintosh 

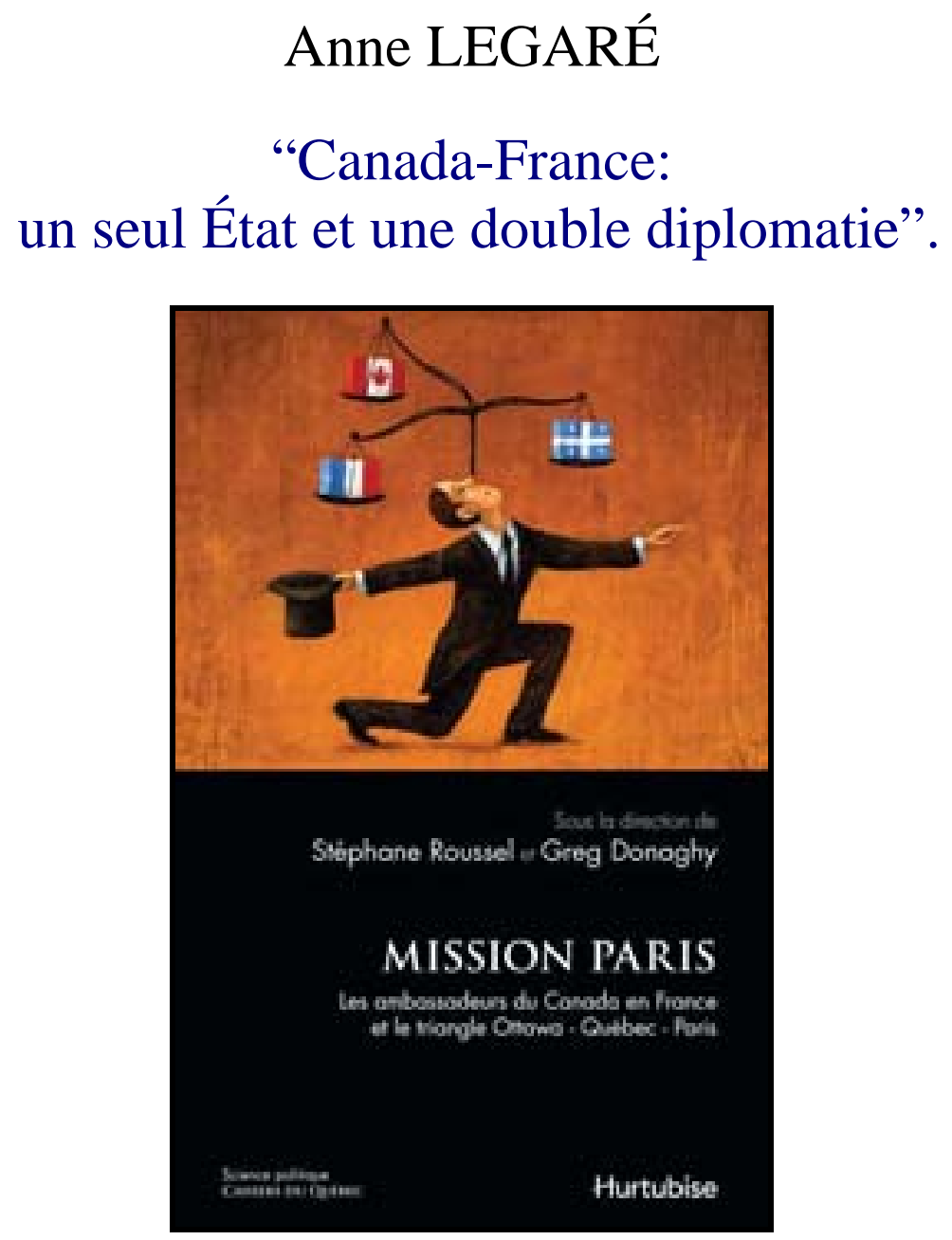

Un chapitre publié dans l'ouvrage sous la direction de Greg Donaghy et Stéphane Roussel, Mission Paris. Les ambassadeurs du Canada en France et le triangle Ottawa-Québec-Paris, chapitre VIII, pp. 163-176. Montréal : Les Éditions Hurtubise, 2012, 216 pp. Collection : Cahiers du Québec, science politique. 


\section{Table des matières}

D’un côté, un vaste champ d'action mais une autonomie inexistante

Canada-France : une relation emblématique

$\underline{L}$ ’État en régime fédéral et les confusions du langage constitutionnel

La reconnaissance internationale, recours pour la légitimation de l’État

$\underline{\text { Un conflit traduit dans un récit officiel }}$

Les tensions d'une double diplomatie

Pourtant, le consentement du Québec prend des formes multiples...

$\underline{Y}$ a-t-il lieu de conclure? 


\section{Anne Legaré \\ “Canada-France : un seul État et une double diplomatie”.}

Un chapitre publié dans l'ouvrage sous la direction de Greg Donaghy et Stéphane Roussel, Mission Paris. Les ambassadeurs du Canada en France et le triangle Ottawa-Québec-Paris, chapitre VIII, pp. 163-176. Montréal : Les Éditions Hurtubise, 2012, 216 pp. Collection : Cahiers du Québec, science politique.

Plus que jamais résolu à englober la relation diplomatique que le Québec entretient avec la France, le Canada cherche depuis de Gaulle à soumettre l'autonomie du Québec à la domination de sa politique étrangère. Certains événements récents, comme les célébrations du 400e anniversaire de la ville de Québec que le Canada a partagées avec la France au cours de 2008, ont été l'illustration éloquente de ce projet structurant : un partenariat diplomatique entre le Canada et la France profitable à l'unité canadienne, c'est-à-dire une alliance visible, coordonnée, maîtrisée et fructueuse dans laquelle la relation du Québec avec la France passerait de son statut de relation privilégiée et directe pour devenir soumise à cette diplomatie d'ensemble.

\section{D'un côté, un vaste champ d'action mais une autonomie inexistante}

Retour à la table des matières

Or, jusqu’à aujourd’hui, ce ravalement diplomatique du Québec n’a pas été si facile à réaliser. Compte tenu des structures juridico-constitutionnelles du Canada inscrites dans un modèle de fédéralisme inachevé et compte tenu de l’histoire des relations fédérales/provinciales, le gouvernement central, même s’il ne peut en restreindre totalement le champ d'action, est confronté aux avancées du Québec en matière de relations internationales. 
[164]

L'autonomie du Québec ou de toute province est cependant quasi inexistante, même dans les domaines de sa propre juridiction, dans cette forme de régime qu'est le fédéralisme canadien. Si la doctrine Gérin-Lajoie (qui a seulement le statut de doctrine et qui est contestée par Ottawa) stipule que les provinces disposent d'un champ d'action internationale légitime dans les domaines de leurs compétences, ce type d'action ne se fait pas sans être soumis à la surveillance du gouvernement fédéral. Le champ d'action peut être vaste, mais l'autonomie de fait reste une vue de l'esprit. C'est pourquoi, en conclusion à une comparaison entre le fédéralisme canadien et le projet de fédéralisme européen, la juriste Andrée Lajoie a été amenée à une étonnante anticipation : « L'éventualité la plus probable est en effet celle de la disparition plus ou moins lente du fédéralisme qui achève de se dissoudre dans une trajectoire où la constitution canadienne s'oriente de plus en plus irrémédiablement vers le monisme d'un État unitaire ${ }^{1}$. »

Le fédéralisme, comme forme de régime, tout en cédant aux régions fédérées (qui disposent de gouvernement mais ne sont pas des États au sens du droit international) une pleine ou relative autonomie dans l'exercice des pouvoirs qui leur incombent, est confronté à cette instance de légitimité ultime qu'est l'État. L’État, qui ne se confond pas avec les paliers de gouvernement, agit sur un territoire donné comme autorité légitime, ce qui lui permet d'être pleinement reconnu comme sujet du droit international. Cette obligation de subordination des instances fédérées (ici, les provinces) par rapport à cette légitimité ultime permet à l’État, entité abstraite, de constituer un ensemble politique, incarné à travers un bloc de dirigeants, qui coordonne, régularise, impose une cohérence plus ou moins étendue (comme on le voit à travers les divers cas d'espèce, États-Unis, Allemagne, Belgique, Iraq, etc.) afin que chaque espace de pouvoir s'ajuste aux processus nécessaires pour la survie de l'ensemble en tant qu'ensemble, ayant seul les prérogatives de l'État (armée, tribunaux de dernière instance, fiscalité, pouvoir résiduel, etc.). C'est dans ce cadre que le Canada, en tant qu'État et tentative de synthèse inachevée et inachevable, [165] s'efforce de soumettre à son autorité les conquêtes de pouvoir d'une de ses régions, le Québec, reconnu, qui plus est, comme nation.

1 Andrée Lajoie, Le fédéralisme canadien : science politique fiction pour l'Europe, conférence prononcée à l'Université d'été de Montpellier, 8 juillet 2004, p. 14. 


\section{Canada-France : une relation emblématique}

$\underline{\text { Retour à la table des matières }}$

À cet égard, aussi curieux que cela puisse paraître, la relation du Canada avec la France est devenue emblématique de cette obligation de cohésion qui pèse sur le Canada en tant qu'État, malgré les références avortées aux pouvoirs que confère le fédéralisme (et/ou la constitution) aux entités fédérées. En effet, ce modèle de fédéralisme est, depuis son origine, le symptôme d’une difficulté à intégrer le Québec dans cet ensemble, c'est-à-dire à consolider la légitimité pleine et entière du Canada sur cette nation/région (dit autrement, à soumettre le Québec à sa seule légitimité). Aux yeux d’Ottawa, les avancées autonomistes du Québec en matière de relations internationales, obtenues grâce à une relation privilégiée avec la France, représentent une dissonance dans la recherche de cohésion du Canada, dans la mesure où elles agissent comme puissance symbolique dans la construction de l’identité québécoise.

La relation du Canada avec la France est donc non seulement stratégique dans la mesure où les deux États sont des alliés (ou des «partenaires ») au sein de rapports mondiaux complexes, mais elle se veut, pour le Canada, un facteur clé dans sa relation de domination et de contrôle d'un Québec animé par une grande volonté d'autonomie, de reconnaissance et d'indépendance. C'est ainsi qu’on a pu observer, au fur et à mesure que le Québec affirmait son identité politique, que la légitimité de la domination du Canada sur le Québec (ou l'effet de synthèse recherché) ne souffrait plus d’être questionnée.

À l'étude des relations diplomatiques de la France avec le Canada telles qu'on peut les explorer dans les archives diplomatiques du Quai d'Orsay, on constate l'évidence de cet irritant pour Ottawa qui n'a cesse de demander à la France la reconnaissance manifeste de sa centralité en tant qu’État, demande que la France a tenté malgré tout de ménager depuis cinquante ans. Il aura fallu une conjoncture politique nouvelle, l'arrivée au pouvoir d'un président aux ambitions de rupture, Nicolas Sarkozy, pour que la [166] demande canadienne soit manifestement entendue. Dans l'intention d'Ottawa et de ses représentants diplomatiques en Fran- 
ce, le soutien direct de la France à un nouveau partenariat avec le Canada, déplaçant de façon manifeste la relation privilégiée du Québec, pourrait se vouloir la condition d'une re-canadianisation du Québec, soit d'un retour à la primauté de l’identité canadienne sur l'identité québécoise. Dans cet esprit, à travers la mise en scène de cette nouvelle symbolique de la relation de la France reconnaissant ses liens avec le Canada et accablant ainsi du même coup le couple QuébecFrance de sa prééminence, Ottawa poursuit sa quête d'une identification plus forte des Québécois à l’entité politique qu'est le Canada.

\section{L'État en régime fédéral et les confusions du langage constitutionnel}

$\underline{\text { Retour à la table des matières }}$

Ce texte ne propose pas une description de cette relation. Il ne consiste pas à rappeler encore une fois les nombreux exemples qui renforceraient la proposition à l'effet que, dans la pratique de ses relations, le Canada a suffisamment démontré son désir de subsumer les relations du Québec avec la France à sa propre diplomatie. Il ne s’agit pas non plus d’évoquer les gains obtenus de haute lutte par le Québec dans des conditions adverses, car de nombreux chercheurs l'ont déjà fait brillamment. Mais dans le cadre d'une réflexion plus particulièrement consacrée aux relations triangulaires Canada-Québec-France de 1945 à 2009, ce qu'il convient d'appeler les tensions d'une double diplomatie, on pourrait s'attendre à ce que ce texte s'attarde sur les dysfonctionnements ou le fonctionnement de ces relations. En l'occurrence, il pourrait être tentant de montrer comment le Canada se comporte comme le mari trompé, tentant sans relâche de ramener l'épouse dans les rets du foyer conjugal.

Ce texte s'applique plus modestement à tenter d'expliquer ce qui fait la trame de fond de cette jalousie inextricable dont souffre le Canada à l'endroit des relations du Québec avec la France. Car les acteurs politiques qui se donnent pour obligation de guérir le Canada et le Québec de cette maladie ont aussi le devoir de se pencher sur toutes les causes du mal qu'ils veulent soigner. On pourrait dire, sans faire de jeu de mots, que ce mal provient pour [167] une part du mésusage 
d'un mot: chaque province ou chaque gouvernement provincial se prenant ou étant pris pour un «État ».

La jalousie qu'éprouve le Canada à l'égard des liens du Québec avec la France provient en grande partie, selon l'analyse qui suit, de l'usage abusif par chacun du mot État pour désigner une province. Ce mésusage est introduit dans le débat politique par l'emploi constant du langage juridique ou constitutionnel propre au fédéralisme au détriment d'une représentation appropriée des enjeux politiques sous-jacents. Les représentations nécessaires pour désigner ce qui appartient au politique, les rapports de pouvoir, le gouvernement, l'État, ne désignent pas des objets identiques lorsque ces mêmes mots sont mis en oeuvre dans le discours juridique. Il en va tout particulièrement ainsi de l'usage du mot État. Pensons par exemple à la substitution du mot État à la place de celui de province ou région fédérée pour désigner les ordres de gouvernement et la répartition de leurs compétences réciproques, mésusage emprunté au langage constitutionnel entraînant les conséquences et les déformations multiples que l'on sait.

Ce texte formule l'hypothèse que le langage constitutionnel qui gouverne nos représentations du politique au Canada et au Québec (en utilisant l'expression d’États fédérés et de partage des compétences) occulte une lutte profonde de légitimité de la domination d'un palier de gouvernement, le palier fédéral ou central, qui se comporte comme s’il était le seul détenteur de la légitimité étatique. Inversement, le Québec, région et nation fédérée ${ }^{2}$, appelée incorrectement «État provincial », revendique dans le champ de ses compétences la pleine et entière autonomie d'un État.

Pour renforcer cette démonstration, il est utile de faire un détour par la pensée d'un auteur libéral du XXe siècle, Max Weber, un des principaux fondateurs d'une pensée véritablement politique de l'État. Dans son petit opuscule et néanmoins classique Le savant et la politique, paru en 1919, Weber a fourni une définition de l'État susceptible d'éclairer le présent siècle, quitte à être appliquée à des ensembles plus larges que ceux qui sont réunis [168] actuellement par les re-

2 Il s'agit ici de la poursuite d'une réflexion sur le concept de région depuis la publication d'un article paru en 1985 ; Anne Légaré, La décentralisation : pourquoi faire? "Quelques courants dominant l'analyse de la région en France et propos sur la définition de la région », $C a$ hiers de sociologie, Montréal, Département de sociologie de l’UQAM, vol. 3, no 1, avril 1985, p. 54 à 73. 
lations régionales ou même continentales. En préface à la réédition de l’ouvrage de Weber, Raymond Aron écrivait que « la science qu’il [Weber] conçoit est celle qui est susceptible de servir l'homme d'action », car, ajoute Aron, si l'attitude de l'homme d'action « diffère en sa fin, elle ne diffère pas en sa structure, de celle de l'homme de science 3 ». Il n'est donc pas utopique d'espérer que cette préoccupation scientifique éclaire aussi les relations Canada/Québec.

Weber définit ainsi l’État :

L'État consiste en un rapport de domination de l'homme sur l'homme fondé sur le moyen de la violence légitime (c’est-à-dire sur la violence qui est considérée comme légitime).

L’État ne peut donc exister qu'à la condition que les hommes dominés se soumettent à l'autorité revendiquée chaque fois par les dominateurs ${ }^{4}$.

Weber explique que pour que cette violence, moyen spécifique de l’État, soit considérée comme légitime, le politique fait appel à trois « raisons internes à la domination » pouvant la justifier et constituant en même temps les trois fondements de sa légitimité. Outre les deux premiers fondements qui sont le pouvoir transmis par la tradition et celui qui est engendré par le charisme, la domination de l'État est reconnue par les hommes comme légitime lorsqu'elle «s'impose en vertu de la légalité, en vertu de la croyance en la validité d'un statut légal ${ }^{5}$ ». Voyons brièvement ce qui pourrait fonder la validité du statut légal de la domination du palier fédéral ou central de gouvernement au Canada dans le champ des relations internationales.

3 Ibid., p. 101-102.

4 Max Weber, Le savant et la politique, Paris, Plon, Union générale d'éditions, 1959, p. 8. Nos italiques. [Livre disponible dans Les Classiques des sciences sociales. JMT.]

5 Ibid. 


\section{La reconnaissance internationale, recours pour la légitimation de l'État}

$\underline{\text { Retour à la table des matières }}$

À l’égard de la légalité du statut du gouvernement fédéral par rapport aux relations internationales, un statut constitutionnel qui lui donnerait l'exclusivité ou l'autorité nécessaire pour fonder « la [169] croyance » (Weber) ou la légitimité en sa prétention légale à être seul en droit de les exercer, le cadre constitutionnel canadien est faible, sinon insuffisant. Le gouvernement fédéral ne peut donc, par les vertus constitutionnelles, faire croire à la validité de sa domination exclusive. À titre d'exemple, on rappelle toujours le jugement du Comité judiciaire du Conseil privé à Londres qui créa un précédent en stipulant que l'article 132 de l'Acte de l'Amérique du Nord britannique confère le pouvoir de conclusion des traités internationaux à l'exécutif, tout en attribuant aux provinces, appelées « les paliers législatifs », en vertu de l'article 92 qui porte sur les domaines de compétences provinciales, leur mise en oeuvre (soit l' «exécution de ces obligations ») ${ }^{6}$. Ainsi, estimant disposer de la reconnaissance de son droit exclusif en matière de conclusion des traités (ce qui en fait un sujet du droit international), le gouvernement fédéral justifie ainsi son constant souci de soumettre toute activité internationale à sa surveillance et à son autorité la plus étroite, sans vraiment réussir à en convaincre le Québec. Le cadre juridique ou constitutionnel donné par le fédéralisme canadien ne suffit donc pas à asseoir solidement la croyance en la légitimité de la domination du gouvernement fédéral en matière de relations internationales et, du même coup, la croyance en son rapport ultime et exclusif à la fondation de l'État. L'État canadien existe pourtant et il n'est pas réductible à l'addition des pouvoirs des gouvernements des provinces plus ceux du gouvernement central, non plus qu'à l'une ou l'autre province appelée incorrectement «État ».

6 Voir, entre autres, l'arrêt de Lord Atkins qui a fait jurisprudence en la matière depuis les avis du juge en chef Lord Lyman Duff (au cours de ses fonctions, 1933 à 1944), cité et contesté de multiples manières dans Jacques-Yvan Morin, Francis Rigaldies et Daniel Turp, Droit international public, 3e éd., Montréal, Thémis, 2001. 
Le Canada doit donc faire appel, pour fonder la légitimité de sa domination, à la reconnaissance des conditions extérieures de celle-ci, plus particulièrement à son statut d’État au sens du droit international. Le rappel de la reconnaissance extérieure de sa légitimité, comme instance ultime de domination sur un territoire, par tout autre État, en l'occurrence les États-Unis et la France, renforce cette légitimité, sans quoi elle apparaît contestable et est [170] contestée. La reconnaissance symbolique du Canada par la France, entendue fortement à travers les paroles du président français Nicolas Sarkozy à l'occasion de sa venue à l'Assemblée nationale du Québec le 17 octobre 2008, puis lors de la remise de la Légion d'honneur au premier ministre du Québec Jean Charest le 2 février 2009, constitue un mode de restauration et de légitimation de la puissance et de la domination du Canada sur une de ses régions fédérées, le Québec, sorte de ravalement au statut de province, envers et contre les acquis d'une identité construite depuis les trente dernières années. Pour dépasser les limites du langage juridicoconstitutionnel du fédéralisme et produire l'effet révélateur du langage proprement politique, c'est-à-dire pour donner son plein sens au mot État, la reconnaissance internationale du Canada en tant qu’État aura été indispensable. La démonstration aura ainsi été effectuée que le Québec, en tant que province, n'est ni un État fédéré ni un État tout court. C'est dans cet esprit, d'ailleurs, que les autorités diplomatiques françaises ont qualifié leur relation avec le Québec de «sujet indirect de la diplomatie française 7 ».

\section{Un conflit traduit dans un récit officiel}

$\underline{\text { Retour à la table des matières }}$

Inutile de faire une nouvelle démonstration de la réalité de ce conflit : on en trouve même l'illustration sur le site du ministère des Affaires étrangères dans une

7 Le sujet direct étant bien entendu le Canada au sens de sujet de droit international. Voir la longue enquête effectuée auprès des autorités françaises à ce propos dans Anne Legaré, Le Québec, otage de ses alliés. Les relations du Québec avec la France et les États-Unis, Montréal, VLB, 2003, ch. VI, « Les intérêts de la France à l’endroit du Québec », p. 187-210. 
section concernant l'historique de son action, plus particulièrement rappelée à l'occasion des célébrations de son Centenaire ${ }^{8}$.

On peut ainsi y lire :

Au cours de la période 1957-1968: le gouvernement fédéral s’inquiétait du positionnement que le Québec se donnait à l'étranger [...] En 1964, Ottawa négocia un accord-cadre avec Paris autorisant Québec et Paris à signer des ententes de coopération. Toutefois, il devint [171] impossible d'éviter l'affrontement, car de Gaulle s'ingérait de plus en plus dans les affaires canadiennes [...] On atteignit le point culminant lors de la visite que fit de Gaulle au Québec en 1967 [... ] Le Général rentra chez lui, laissant en ruines les relations canado-françaises.

Le ministère en tire une conséquence :

La question de l'unité nationale fut alors inscrite à l'ordre du jour politique du Canada [...] Le dossier de l'unité posa de nouveaux défis et ouvrit de nouvelles possibilités pour la diplomatie canadienne [...]

Pour la période qui suit, de 1968 à 1984, le conflit se resserre :

Trudeau [...] situait au premier rang de ses priorités l'unité nationale, car le sentiment séparatiste gagnait du terrain au Québec vers la fin des années 1960 [... ${ }^{9}$

La question d'ordre intérieur la plus importante au Canada, le statut du Québec, continuait d'avoir des incidences sur la scène internationale [...] 10

[les] gouvernements fédéral et québécois jouèrent des coudes pour se mettre en position avantageuse au moment où le Québec affirmait son droit à une voix indépendante sur la scène internationale lorsqu'on débattait de questions de compétence provinciale. De son côté, Ottawa insistait pour que les affaires étrangères demeurent le domaine exclusif du gouver-

8 Jouer dans la cour des grands. Histoire du ministère des Affaires étrangères et du Commerce international, Ottawa, MAECI, 2008, www.international.gc.ca/department/history-histoire, p. 10-11.

9 Ibid., p. 2.

10 Ici, il est rappelé sur le site les pressions de la France auprès du Gabon afin qu'il invite le Québec à participer à une réunion d’États. 
nement fédéral [...] La question du Québec prit un caractère encore plus aigu après l’élection du Parti québécois, formation séparatiste, en $1976{ }^{11}$.

Dans une autre section on lit :

En 1964, Marcel Cadieux s’oppose farouchement au séparatisme [...] Paul Martin et Marcel Cadieux veillent à ce que le ministère tienne compte des aspirations des Canadiens français [...] À cette fin, ils nouent des liens plus étroits avec la France.

\section{Les tensions d'une double diplomatie}

$\underline{\text { Retour à la table des matières }}$

Quarante-cinq ans plus tard, à l’occasion des propos interventionnistes du président Sarkozy, une déchirure est manifeste dans [172] la symbolique de la relation entre le Québec et la France. Du côté canadien, les ambitions de reconnaissance manifeste de l'unité du Canada par la France, tentatives fédérales bien connues depuis les années 1960, ont été pleinement satisfaites.

Cependant, on peut se demander s'il est normal que deux paliers de gouvernement d'un régime dit fédéral s’affrontent pour obtenir de façon privilégiée ou exclusive les bonnes grâces d'un État-tiers, la France. Comment analyser ce qui fonde cette déchirure du consensus dans la relation Québec-Canada, à l’intérieur d’un régime fédéral prétendument décentralisé, à partir du fait que le Québec ait entretenu depuis 1964 et jusqu'en 2008 des relations autonomes avec la France liées à sa langue et à sa culture ?

Afin d'expliquer cette tension, à la fois d'ordre structurel et conjoncturel, il est utile de préciser la nature de divers recours symboliques à la domination de l’État en régime fédéral.

D’abord, tout État, comme le rappelle Weber, est un rapport de domination sur une population et sur un territoire qui repose sur le consentement de ceux qui y sont soumis, renforçant ainsi la cohésion de l'ensemble et fondant sa légitimité. Dans un régime fédéral, cette domination s'exerce par le relais des gouverne-

11 Op. cit., p. 7. 
ments du centre et des régions et le consentement des populations des régions fédérées ; dans un régime unitaire, ce consentement est obtenu autour de la centralité des institutions et d’une configuration unificatrice du pouvoir.

Dans les sociétés contemporaines, cette domination appuie sa légitimité sur un ordre légal ${ }^{12}$. Au Canada et au Québec, c'est dans le recours au discours ou au langage constitutionnel portant sur le fédéralisme en général et sur le fédéralisme canadien en particulier que s'exerce la légitimation de la domination de l'État sur l'ensemble des territoires et des populations (sur les citoyens et sur les provinces) du Canada. Mentionnons, à titre d’exemple, les références constantes dans le débat politique aux théories du fédéralisme, à la répartition des compétences, aux catégories juridiques comme ordre ou palier de gouvernement, aux pouvoirs du gouvernement fédéral vis-à-vis des compétences des gouvernements provinciaux, à la jurisprudence en matière de relations fédérales/provinciales, etc.

Or, dans le cas où le consentement à cette domination est remis en question par une population fédérée, l’État, à travers ses représentants, aura recours à toute forme de violence symbolique, perçue comme légitime ${ }^{13}$, afin de réinstaurer la cohésion menacée. Les différents recours du gouvernement fédéral à la reconnaissance internationale de l'unité nationale du Canada agissent en vue de restaurer cette domination légitime de l'État sur le Québec en particulier, aussi bien que sur l'ensemble.

De façon concrète, ces recours peuvent prendre les formes suivantes :

a) la recherche d'unité nationale, envers et contre les prétentions décentralisatrices du fédéralisme ;

b) dans l'exercice de la légitimité de sa domination, l’État, par la voix des gouvernements, aura recours à toute violence, qu'elle soit symbolique, légale ou physique, comme, par exemple, le recours à l'autorité des gouvernants, la soumission à la loi et aux tribunaux, le prélèvement des impôts, etc. Et, faisant usage des relations internationales à ses propres fins, l’État

12 Plutôt que sur la tradition ou sur le charisme des dirigeants.

13 À titre de contre-exemple, on peut penser, comme l’a révélé la Commission Gomery, au scandale des commandites. 
exercera sa domination par diverses formes de coordination de l'action des provinces avec le gouvernement fédéral (que sont, par exemple, les avis de pertinence demandés par les fonctionnaires des gouvernements provinciaux à l'instance fédérale ou par la soumission des représentants des provinces aux règles de la Convention de Vienne, etc.) ;

c) enfin, l'État pourra avoir recours à d'autres sujets de droit qui incarnent les conditions de sa légitimité. C'est à ce propos que les relations du Québec avec la France, suivant le cours de la tendance autonomiste instituée par la France depuis de Gaulle, consacrant ainsi la reconnaissance d'une double diplomatie (quoique le Québec en soit demeuré le «sujet indirect »), ont représenté le point sensible, le maillon faible dans la capacité du Canada comme État à assurer sa domination sur l'ensemble et en particulier sur le Québec. En effet, toutes les manifestations de reconnaissance du Canada par la France, même symboliques, représentent des éléments constitutifs de la légitimité du [174] Canada dans sa domination de l'ensemble, y compris le Québec, faut-il le préciser. Si on peut dire que la France n'a pas manqué à cette mission depuis de Gaulle, il reste qu'elle a pu s'en éloigner aussi de diverses manières, plus ou moins discrètes, plus ou moins officielles, de telle sorte que le Canada n'a eu de cesse de tenter de la rappeler à sa reconnaissance. Les récentes déclarations du président Sarkozy semblent vouloir mettre fin à cette valse-hésitation.

\section{Pourtant, le consentement du Québec prend des formes multiples...}

$\underline{\text { Retour à la table des matières }}$

Retournons maintenant vers l'histoire de cet enjeu, car les différents acteurs canadiens et québécois, au cours des presque cinquante dernières années, ont mené ces luttes de pouvoir bien différemment. Il serait injuste de subsumer l'ensemble des positions souverainistes à une véritable vision sécessionniste du Canada. De plus, du point de vue de la stratégie et de la tactique, les dispositions devant l'unité du Canada varient ; de la même manière, les théories évoquées ont 
plutôt tendance à consentir et donc à reproduire, quoique sous diverses formes, la domination du Canada sur l'ensemble de ses territoires et des populations. 
1) Du point de vue du Québec d'abord, des voix discordantes ont mené le projet de souveraineté. S’il est vrai que la souveraineté du Québec a nourri auprès de certains l'idée d'une véritable rupture avec le Canada ${ }^{14}$, l'échelle des disparités dans la conception de ce projet est assez vaste pour que l'on admette au moins que l'heure actuelle est plutôt à une mise de côté d'une conception portant atteinte à l'intégrité du Canada, c'est-à-dire à une conception pleine et entière de la souveraineté, telle que certains la qualifieraient de pure et dure !

2) D’autres s'appuient plutôt sur ce que Weber appelle la légitimité de l'ordre légal donné par le fédéralisme canadien pour exercer, valoriser ou revendiquer l'extension des relations internationales du Québec dans le cadre même des relations fédérales-provinciales. Ces défenseurs vont de Paul Gérin-Lajoie à Jean Charest en passant par Gilles Duceppe et par tant d'autres souverainistes : tous affirment la compatibilité des relations internationales du Québec avec la domination du Canada sur l'ensemble des provinces et ils en poursuivent le développement dans ce contexte.

3) Sur le plan de l'analyse politique, des théories subtiles et séduisantes reflètent aussi cette logique de domination de l'État en s'appuyant sur les représentations les plus courantes du fédéralisme : ce sont celles qui, empruntées au droit constitutionnel, font appel aux notions d’État fédéré pour parler des gouvernements provinciaux, de paradiplomatie ou de souveraineté partagée entre ordres de gouvernement pour désigner un régime politique de partage des pouvoirs qui n'est autre que l'organisation spécifique de la domination de l'État au Canada. Toutes les théories reconnaissent le pouvoir ultime de cet État, qui se trouve bien quelque part, puissance indivisible et invisible, et que tous sont enclins à honorer.

14 Cette affirmation vaut en tant que tendance même si, au niveau des acteurs et des promoteurs de ce processus d'autonomisation, la conscience n'est pas claire ni la responsabilité complète à l'égard des effets attribuables à la scission du Canada. Par ailleurs, beaucoup de souverainistes souhaitent avant tout une refondation des rapports entre le Québec et les autres provinces du Canada qui n’aurait pas pour effet une complète séparation. 


\section{Y a-t-il lieu de conclure?}

Retour à la table des matières

On l'a rappelé, le champ d'action des provinces peut être vaste, mais leur autonomie est quasi inexistante, compte tenu du fédéralisme canadien qui subordonne chaque province à la logique unitaire de l’État. C'est pourquoi, dans un tel contexte, la reconnaissance par la France de la spécificité culturelle du Québec pèse de tout son poids en agissant comme catalyseur d'une identité francophone sans arrêt fragilisée. En retour, le Canada n’a de cesse de vouloir consolider son autorité à l’intérieur d'un régime fédéral qui déguise sa domination. Afin de l'exercer selon les apparences de la légitimité, le Canada a recours à la scène internationale et comme on l'a vu dans l'actualité récente, à l'autorité du chef de l’État français, qui a exprimé sans réserve son soutien à l’unité canadienne.

Le dernier chapitre des relations entre le Canada et la France soulève à ce propos quelques questions. Le président Sarkozy, à la suite d'une allocution à l’Assemblée nationale du Québec à l'occasion de sa présence au Sommet de la Francophonie le 17 octobre 2008, s'est prêté à une conférence de presse au cours de laquelle il a clairement affiché sa préférence pour l'unité du Canada. Lors de la remise de la Légion d’honneur à Jean Charest, à Paris, le 2 février 2009, il a réitéré cette position.

Nicolas Sarkozy a ainsi fait usage de la violence symbolique d'État en tant que président d'un État étranger, la France, particulièrement cher aux Québécois. Cette violence symbolique, utilisée par le président Sarkozy s’adressant à eux pour leur parler de leur destin dans le Canada, s’est présentée comme une intervention directe en faveur de l'unité canadienne, sans ménagement pour les aspirations politiques de la moitié des Québécois.

Cette intervention suggère deux interprétations : 1) soit le président français s'est cru inconsciemment en territoire français et en droit d'intervenir légitimement afin d’infléchir les représentations de ces citoyens ; 2) soit Nicolas Sarkozy a voulu rappeler aux Québécois la primauté du Canada sur le Québec par le re- 
cours à cette violence symbolique. Ce faisant, il s'est placé sur le terrain de la reconnaissance internationale, instance légitime pour rappeler la domination du Canada en tant qu'État.

Cette leçon pourra avoir deux effets à travers le temps : 1) soit elle atteindra le but poursuivi en contribuant à une re-canadianisation du Québec, en renforçant le consentement et le sentiment d'identification des Québécois au Canada ; 2) soit cette intervention conduira les Québécois à mieux saisir que seul le statut d’État pourrait les protéger contre les autres États, fussent-ils des alliés, qui, poursuivant leurs propres intérêts, ont pour effet de soumettre le Québec et d'en faire l'otage passif de leurs intérêts.

\section{Fin du texte}

